\title{
Surveying the Bright Sky
}

\author{
Arne A. Henden \\ AAVSO, 49 Bay State Road, Cambridge, MA 02138 \\ email: arne@aavso.org
}

\begin{abstract}
The AAVSO is initiating a new survey of the sky. It will cover the entire visible sky, both north and south, on a daily basis, in two colours, and with a limiting magnitude of $V=17$. This will be a perfect complement to LSST, but will be available years earlier and will continue into the indefinite future. The photometry will be publicly available within 24 hours through our website. Some details of the hardware and operations are described.
\end{abstract}

Keywords. surveys, techniques: photometric, stars: variables: other

\section{Introduction}

It would be wonderful if we had continuous coverage of the night sky, with multispectral information about every star visible to the faintest limits and with nearly infinite time-sampling. This utopian goal cannot be reached with modern technology, so any survey will only partially fill the parameter space. Perhaps not surprisingly, not many surveys have so far attempted to pursue that goal. Tycho, the star-mapper instrument on-board the HIPPARCOS satellite (ESA 1997), covered the entire sky during the 4-year mission, with $B_{t}$ and $V_{t}$ photometry down to $9^{\text {th }}$ magnitude per observation $(3 \sigma)$. An average of 130 data points were acquired over the 3.5-year mission for 481553 objects.

The All-Sky Automated Survey (ASAS; Pojmanski 2002) started as a southern system at Las Campanas Observatory in 2000. It covers the southern sky (to Dec $+28^{\circ}$ ) from $6.5<V<14 \mathrm{mag}$; it has $15^{\prime \prime}$ pixels and makes approximately one visit every $2-3$ days. The survey states that it is being done at $V$ and $I_{c}$, though the $I_{c}$ measurements have never been released publicly. A northern site has been operational since 2006, but only one paper (on KEPLER field variables) has been released (Pigulski et al. 2009), and no public data release has been made. No data have been available from the southern site since 2009, and a disk failure a few years ago resulted in a total loss of data for some time.

All other surveys only cover a portion of the night sky, or have other limitations. The wide-field exoplanet surveys like XO or SuperWASP use unfiltered CCDs. PTF and Pan-STARRs only cover either small sections of the northern sky, or only one filter, or only every few nights, and have saturation magnitudes around 14. The Catalina Sky Survey uses a northern and a southern telescope (though most of the current data come from the north), it stays away from the Galactic plane, and is only unfiltered. Future proposed surveys like LSST have saturation limits of $17^{\text {th }}$ magnitude, only cover the sky in one passband at a time, return to a given field on perhaps a weekly basis, and have fixed locations (LSST at Cerro Pachon). The costs of these projects range from $\$ 100 \mathrm{~K}$ to $\$ 400 \mathrm{M}$ just for the hardware, not counting the many man-years of programming effort and the continued operations cost at each site.

The AAVSO has begun an ambitious survey of the night sky to fill in the parameter space between wide-field telephoto lens surveys like ASAS, and the $\$ 100 \mathrm{M}$-class surveys 
using fixed telescopes in the north and south. This survey, the $2^{\text {nd }}$ Generation All-Sky Survey (2GSS), is described below.

\section{Overview}

2GSS is designed to fill a limited parameter space, namely:

- all-sky coverage

- cadence once per night

- dynamic range $10<V<17$

- spatial resolution around $2^{\prime \prime}$

- at least two simultaneous filters

- continuous operation for the indefinite future

- immediate public access to the database

All-sky cover is accomplished through the use of multiple sites around the world. A minimum of two sites would be required for north/south coverage. Owing to expected weather problems and the actual single-system coverage, we will install several systems in both hemispheres. We feel that all-sky is an important aspect, as we are primarily interested in variable-star monitoring, and several rare classification prototypes are found in each hemisphere. At the same time, transient discoveries are indiscriminant regarding their location.

While previous surveys have provided weekly or daily cadence over a small region of sky, we wish to cover the available night sky every 24 hours. Allowing for the solar and lunar avoidance regions, that amounts to about 30,000 square degrees to be covered nightly. It ensures that any variable stars with periods much longer than one day will have their light curves well covered; shorter-period stars with known period will have good coverage over multiple cycles; transient objects will be detected within one day of outburst or decline.

Tycho covered most stars down to about 9-10mag. ASAS covers down to 14 mag, though with blending through the Galactic Plane. LSST saturates at 17 mag. We've decided that the optimal magnitude range is $10-17$, to mesh with the faint end of Tycho and the bright end of LSST, and also to provide 3 magnitudes of extended range beyond ASAS.

One of the biggest problems with ASAS is the $15^{\prime \prime}$ pixel size, which generally means that objects closer than $30^{\prime \prime}$ are measured as one. This blending causes problems in the Galactic Plane, when several stars are often contained within the aperture, and deciding which is the variable, and what is its true amplitude, is difficult. It only gets worse as you go fainter and more stars become visible. For that reason, we have opted to go with finer pixel resolution at the expense of field coverage per image, and to increase the number of systems and the cadence to recover the field coverage.

For deriving knowledge of the type of object being studied, having two or more simultaneous pass-bands of information helps enormously. You then know whether an object is red or blue, you know whether the variation has the same amplitude at both wavelengths, and you can discriminate against cosmic ray and other defects by imaging the same field with two separate systems. These are particularly important for transients, and such a survey will give valuable hints as to how to conduct the far more ambitious LSST survey.

We are committed to providing ready access to all of the data from the 2GSS survey. The standard photometry will be available within 24 hours, and we are planning to release the transient events within a few minutes after an exposure is completed. Longevity is 
equally important. The AAVSO database of observations dates back over 100 years, and our new survey is a key element of our future plans. We intend it to be a dynamic project, changing as technology improves but continuing into the indefinite future.

\section{Hardware Description}

We are pushing commercial technology as much as possible, while remaining costeffective. We anticipate 5 "nodes" in the final configuration: 3 in the north as a hedge against poorer weather, and 2 in the south. We are using seed funding from the Robert Martin Ayers Sciences Fund to develop the first of the northern nodes, to be sited in southern Arizona. Each node will cover about 8,000 deg. ${ }^{2}$ per night, and consists of two telescope/camera systems, either on a single mount or on two smaller mounts. Since this one is a prototype system, we are trying out several configurations of telescopes and mounts to find the optimal solution. Each node will have, inside a single enclosure,

- telescope: (2) APM305 305-mm aperture, f/2.8 focal ratio; corrected prime focus

- camera: (2) FLI ML16803; liquid cooled, $4096 \times 4096$ pixels, 9- $\mu$ pixels.

- filter holder; one filter per telescope, either $g^{\prime}$ or $i^{\prime}$

- Paramount MX mount, one per telescope

- single linux computer running two Virtual Machines, one for each telescope system.

The telescope (seen in Fig. 1) is a unique design that uses a Keller corrector to cover the entire sensor with minimal vignetting and distortion. Since it is prime focus, we chose the Microline camera body for its small size and minimal obscuration. The FLI cameras also download in just a few seconds, minimizing the dead-time between exposures. Such a node, consisting of two telescopes and their mounts, costs $\$ 120 \mathrm{~K}$. Since they are small installations, we anticipate being able to locate the nodes on the grounds of existing observatories with minimal support costs. They are also small enough that they can be moved to other sites if necessary in later years.

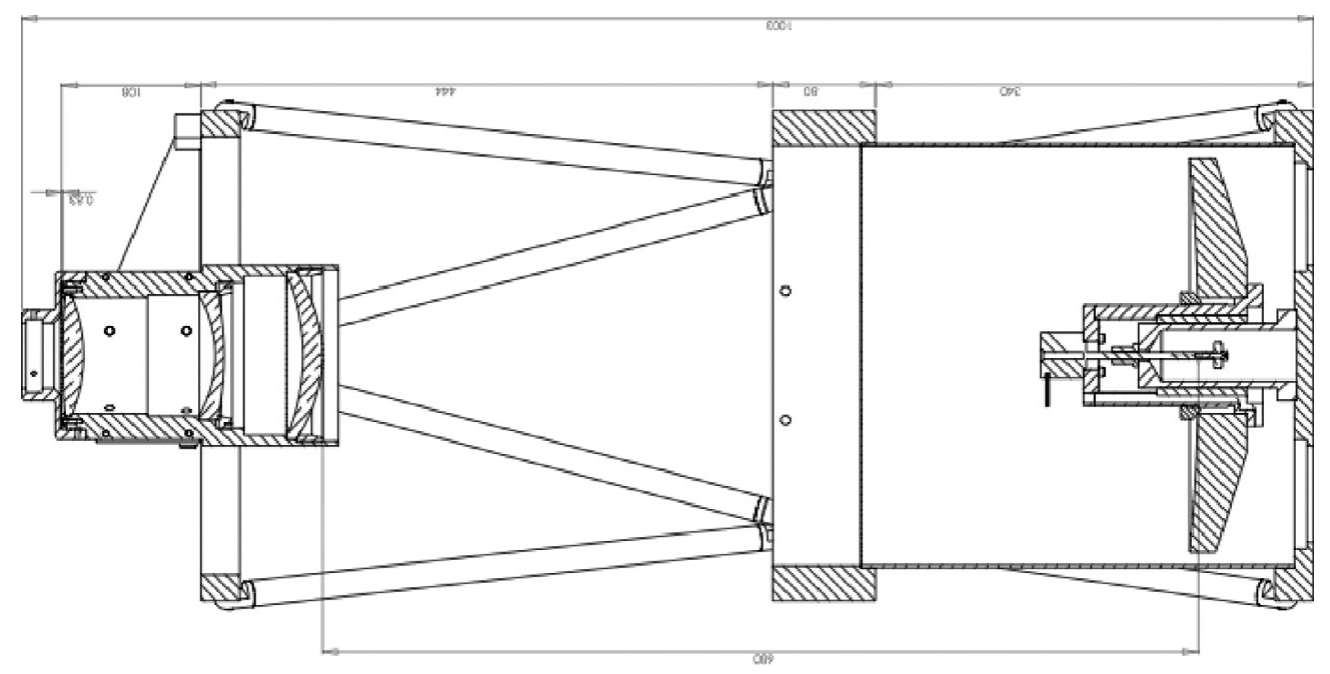

Figure 1. CAD drawing of the APM305 telescope. 


\section{Planned Operation}

According to experimental data from our existing APASS system, which uses 20-cm telescopes and the same CCD sensor, we should reach $17^{\text {th }}$ magnitude in about 15 seconds with these telescopes. We then add 5 seconds for download and 10 seconds to move to the next field, so approximately 1000 fields per night can be covered. The system has a focal length of $85.4 \mathrm{~cm}$, a plate scale of $2^{\prime \prime} .17$ per pixel, and a field of view of $2^{\circ} .47 \times 2^{\circ} .47$ $\left(6.12 \mathrm{deg}^{2}\right)$. To cover the $\sim 30,000 \mathrm{deg}{ }^{2}$ of sky visible each night requires 4744 fields. Since each site covers 1000 fields, a total of 5 nodes is required for daily coverage, with the understanding that some fields may miss a night or two depending on weather. If poor weather persists, another node may perform double-duty, giving temporarily lower cadence but preventing long lapses in coverage.

The images are acquired on a linux computer. At the end of the night, a script is run to perform dark subtractions and flat-field each image using master calibration frames. As with our APASS pipeline, the images are then analyzed using Sextractor and DAOPHOT to obtain good aperture photometry, passed through WCS software to obtain good astrometry, and then stored on an external hard drive. Each star-list is then compared with the APASS master catalogue to obtain zero-points, and mean transformation coefficients are used to transform the data. The observations are then stored in a relational database for public retrieval. From our experiences with APASS, we anticipate this processing to take about 8 hours, so it will be completed during daylight before the next observing session.

Only calibrated star-lists will be transmitted from the local node back to a central site (which is currently planned to be an Amazon Cloud instance). The database there will be accessible through a web-based interface. Once a month local technicians will exchange the external hard drive and send the images back to AAVSO headquarters to be archived.

\section{Current Status}

The APM telescope is due to arrive during 2012 January. The FLI camera is already in-house and we have tested it in our electronics lab. We have filters and a Paramount, so by early 2012 we expect to be doing initial tests of a complete system. A 2-telescope node will be installed in southern Arizona during early 2012, with initial results available before the AAS meeting in June. We are submitting grant applications for purchasing a full 5-node system, and are pursuing Memorandum of Understanding agreements with appropriate sites. Our expectations are that we will be covering approximately 20,000 square degrees with a few-day cadence by mid-2012, with full configuration installed and commissioned during 2013. More information will be posted on the main AAVSO web site (http://www. aavso.org) as the survey begins.

\section{References}

ESA. 1997, The Hipparcos and Tycho Catalogues, ESA SP-1200

Pojmanski, G. 2002, Acta Astronomica, 52, 397

Pigulski, A,. Pojmański, G., Pilecki, B., \& Szczygieł, D. M. 2009, Acta Astronomica, 59, 33 